\title{
The Parameterization of All Stabilizing Multi-Period Repetitive Controllers for Time-Delay Plants with the Specified Input-Output Characteristic*
}

\author{
Kou YAMADA ${ }^{* *}$, Makoto KOWADA**, Takaaki HAGIWARA**, \\ Iwanori MURAKAMI**, Yoshinori ANDO**, Tatsuya SAKANUSHI**, \\ Shun YAMAMOTO** and Nhan LUONG THANH NGUYEN** \\ ${ }^{* *}$ Department of Mechanical Systems Engineering, Gunma University, \\ 1-5-1 Tenjincho, Kiryu, Gunma, 376-8515, Japan \\ E-mail: t09801226@gunma-u.ac.jp
}

\begin{abstract}
In this paper, we examine the parameterization of all stabilizing multi-period repetitive controllers for single-input/single-output time-delay plants with the specified inputoutput characteristic. The parameterization is the problem to find all stabilizing controllers. The parameterization of all stabilizing multi-period repetitive controllers for non-minimum-phase plants and that of all stabilizing multi-period repetitive controllers for time-delay plants were solved by Yamada and Satoh. However, using the method by Yamada and Satoh, we cannot settle input-output characteristics easily. Because the input-output characteristic is related to four free parameters. If the parameterization of all stabilizing multi-period repetitive controllers with the specified input-output characteristic, which is the parameterization when low-pass filters are settled beforehand, is obtained, we can specify the input-output characteristic easily. In this paper, we propose the parameterization of all stabilizing multi-period repetitive controllers for time-delay plants with the specified input-output characteristic.
\end{abstract}

Key words : Repetitive Control, Multi Period Repetitive Controller, Time Delay System, Parameterization, Low Pass Filter

\section{Introduction}

In this paper, we examine the parameterization of all stabilizing multi-period repetitive controllers for time-delay plants with the specified input-output characteristic. The parameterization problem is to find all stabilizing controllers for the plant ${ }^{(1),(2)}$. The parameterization of all stabilizing repetitive controllers was studied by Hara and Yamamoto ${ }^{(3)}$. Because the sufficient condition for stability of a repetitive control system is decided as an $H_{\infty}$ norm problem, the parameterization for repetitive control systems is given by resolution into the Nevanlinna-Pick interpolation problem ${ }^{(3)}$. Katoh and Funahashi ${ }^{(4)}$ gave the parameterization of all repetitive controllers for minimum-phase plants by solving the Bezout equation exactly. Because the parameterization in Ref. (4) is not given based on the sufficient stability condition of the repetitive control system, this result is important in the sense that the class of repetitive controllers is more extensive than the class of repetitive controllers given by Hara and Yamamoto ${ }^{(3)}$. However, the parameterization in Ref. (4) has some difficulties remaining. Reference (4) assumed that the plant is asymptotically stable. This implies that they give the parameterization of all repetitive controllers for an asymptotically stable and minimum-phase plant. That is, Ref. (4) does not give the explicit parameterization of all stabilizing modified repetitive controllers for minimum-phase plants. In addition, in Ref. (4), it is assumed that the relative degree of the low-pass filter in the repetitive controller is equal to that of the plant.

*Received 21 July, 2010 (No. 10-0297) [DOI: 10.1299/jsdd.5.248]

Copyright (c) 2011 by JSME 
This implies that Katoh and Funahashi gave the parameterization of all stabilizing repetitive controllers in which the structure of internal model is limited. Yamada and Okuyama overcame these problems and gave the parameterization of all stabilizing repetitive controllers for minimum-phase plants using the parallel compensation technique and exact solution of Bezout equation $^{(5)}$. Yamada et al. expanded the result in Ref. (5) and gave the complete parameterization of all stabilizing repetitive controllers for non-minimum-phase plants ${ }^{(12)}$. In this way, the parameterization of all stabilizing repetitive controllers for the strictly proper plant have been considered.

However, it is pointed out that the modified repetitive control system has bad disturbance attenuation characteristics ${ }^{(7)}$. In order to improve disturbance attenuation characteristic of the repetitive control system, Gotou et al. proposed the multi-period repetitive controller ${ }^{(7)}$. However, the phase angle of the low-pass filter in a modified repetitive controller and that of a multi-period repetitive controller have a bad effect on the disturbance attenuation characteristics $^{(10),(11)}$. Yamada et al. overcame this problem and proposed a design method for multi-period repetitive controllers to attenuate disturbances effectively ${ }^{(12),(13)}$ using the time advance compensation described in Refs. (10), (11) and (15). Using this multi-period repetitive control structure, Steinbuch proposed a design method for repetitive control systems with uncertain period time ${ }^{(16)}$. The parameterization of all stabilizing multi-period repetitive controllers was solved in Refs. (8) and (14). However, the parameterization in Refs. (8) and (14) cannot be applied to time-delay plants. To solve this problem, the parameterization of all stabilizing multi-period repetitive controllers for time-delay plants was solved in Ref. (9).

According to Ref. (9), the parameterization of all stabilizing multi-period repetitive controllers for time-delay plants includes eight free-parameters. Four of those work to specify the low-pass filter in the internal model for the periodic reference input of which the role is to specify the input-output characteristic. The others work to guarantee the stability of control system. However, using the method in Ref. (9), we cannot settle the input-output characteristic easily. Because, we must settle four free parameters mentioned above in order to specify the input-output characteristic. Since low-pass filters in the internal model for the periodic reference input work to specify the input-output characteristic, if the parameterization of all stabilizing multi-period repetitive controllers for time-delay plants with the specified inputoutput characteristic, which is the parameterization when low-pass filters are settled beforehand, is obtained, we can specify the input-output characteristic more easily than the method in Ref. (9).

In this paper, we expand the result in Ref. (9) and propose the parameterization of all stabilizing multi-period repetitive controllers for time-delay plants with the specified inputoutput characteristic. The basic idea of obtaining the parameterization of all stabilizing multiperiod repetitive controllers for time-delay plants with the specified input-output characteristic is very simple. From the control structure of the multi-period repetitive controller, the parameterization of all stabilizing multi-period repetitive controllers for time-delay plants is reduced to that of all stabilizing multi-period repetitive controllers including time-delay for systems without time-delay. The parameterization of all stabilizing controllers for the plant is obtained using the method of Refs.(1) and (2). The parameterization of all stabilizing controllers includes a free parameter, which is stable ${ }^{(1),(2)}$. That is, stabilizing multi-period repetitive controllers for time-delay plants with the specified input-output characteristic can be obtained using the free parameter in the parameterization of Refs. (1) and (2).

This paper is organized as follows: In $\S 2$, the problem considered in this paper is described. In $\S 3$, the parameterization of all stabilizing multi-period repetitive controllers for time-delay plants with the specified input-output characteristic is clarified. Then, it is found that the number of free parameters in the proposed parameterization which must be settled is very small as compared with that in the parameterization of all stabilizing multi-period repetitive controllers for time-delay systems in Ref.(9), because low-pass filters are settled beforehand. This makes it easy to design multi-period repetitive control systems than the 
method in Ref.(9). Therefore, proposed method is very effective for designing stabilizing multi-period repetitive controllers for time-delay systems. In $\S 4$, control characteristics of control system using the proposed parameterization are described. In $\S 5$, we present a design procedure of stabilizing multi-period repetitive controller for time-delay plants with the specified input-output characteristic. In $\S 6$, we show a numerical example to illustrate the effectiveness of the proposed method. Section 7 gives concluding remarks.

Notation

$R \quad$ the set of real numbers.

$R(s) \quad$ the set of real rational functions with $s$.

$R H_{\infty} \quad$ the set of stable proper real rational functions.

$H_{\infty} \quad$ the set of stable causal functions.

\section{Problem formulation}

Consider the unity feedback control system in

$$
\left\{\begin{array}{l}
y(s)=G(s) e^{-s L} u(s)+d(s) \\
u(s)=C(s)(r(s)-y(s))
\end{array},\right.
$$

where $G(s) e^{-s L}$ is the time-delay plant with input time-delay $L>0, G(s) \in R(s)$ is strictly proper, $C(s)$ is the controller, $y(s) \in R$ is the output, $u(s) \in R$ is the control input, $d(s) \in R$ is the disturbance, $r(s) \in R$ is the periodic reference input with period $T$ satisfying

$$
r(t+T)=r(t) \quad(\forall t \geq 0) .
$$

According to Refs. (7), (8) and (9), the multi-period repetitive controller $C(s)$ in Eq. (1) is written by the form in

$$
C(s)=C_{0}(s)+\frac{\sum_{i=1}^{N} C_{i}(s) q_{i}(s) e^{-s T_{i}}}{1-\sum_{i=1}^{N} q_{i}(s) e^{-s T_{i}}},
$$

where $N$ is multiplicity and an arbitrary positive integer, $C_{0}(s) \in R(s), C_{i}(s) \in R(s)(i=$ $1, \ldots, N)$ and $q_{i}(s) \in R(s)(i=1, \ldots, N)$ are low-pass filters satisfying

$$
\sum_{i=1}^{N} q_{i}(0)=1
$$

and $T_{i}>0(i=1, \ldots, N)$ is multi-period. Without loss of generality, it is assumed to hold $C_{i}(s) \neq 0(\forall i=1, \ldots, N)$ and $q_{i}(s) \neq 0(\forall i=1, \ldots, N)$.

From Ref. (7), if low-pass filters $q_{i}(s)(i=1, \ldots, N)$ satisfy

$$
1-\sum_{i=1}^{N} q_{i}\left(j \omega_{k}\right)=0 \quad(\forall k=0,1, \ldots, n),
$$

where $\omega_{k}$ are frequency components of the periodic reference input $r(s)$ written by

$$
\omega_{k}=\frac{2 \pi k}{T}(k=0,1, \ldots, n)
$$

and $\omega_{n}$ is the maximum frequency component of the periodic reference input $r(s)$, then the output $y(s)$ in Eq. (1) follows the periodic reference input $r(s)$ with small steady state error. Using the result in Ref. (9), in order for $q_{i}(s)(i=1, \ldots, N)$ to satisfy Eq. (5) in wide frequency range, we must design $q_{i}(s)(i=1, \ldots, N)$ to be stable and of minimum-phase. If we obtain the parameterization of all stabilizing multi-period repetitive controllers such that $q_{i}(s)(i=1, \ldots, N)$ in Eq. (3) are settled beforehand, we can design the multi-period repetitive controller satisfying Eq. (5) more easily than the method in Ref. (9). Since $q_{i}(s)(i=1, \ldots, N)$ 
work to specify the input-output characteristic of the control system in Eq. (1), we call the parameterization of all stabilizing multi-period repetitive controllers such that $q_{i}(s)(i=1, \ldots, N)$ in Eq. (3) is settled beforehand the parameterization with the specified input-output characteristic.

The problem considered in this paper is to clarify the parameterization of all stabilizing multi-period repetitive controllers for time-delay plants with the specified input-output characteristic. That is, when low-pass filters $q_{i}(s) \in R H_{\infty}(i=1, \ldots, N)$ in Eq. (3) are settled beforehand, we obtain the parameterization of all stabilizing multi-period repetitive controllers $C(s)$ written by Eq. (3) for the plant $G(s) e^{-s L}$.

3. The parameterization of all stabilizing multi-period repetitive controllers for time-delay plants with the specified input-output characteristic

In this section, we give the parameterization of all stabilizing multi-period repetitive controllers $C(s)$ for the plant $G(s) e^{-s L}$ with the specified input-output characteristic.

Low-pass filters $q_{i}(s)(i=1, \ldots, N)$ in Eq. (3) are assumed to be settled beforehand and to be stable and of minimum-phase. The parameterization of all stabilizing multi-period repetitive controllers $C(s)$ for the plant $G(s) e^{-s L}$ with the specified input-output characteristic is summarized in the following theorem.

Theorem 1 The controller $C(s)$ stabilizes the control system in Eq. (1) and works as the multi-period repetitive controller, that is, $C(s)$ is written by Eq. (3), and $C_{0}(s)$ and $C_{i}(s) \neq$ $0(i=1, \ldots, N)$ in Eq. (3) are rational functions if and only if the controller $C(s)$ is written by

$$
C(s)=\frac{X(s)+D(s) Q(s)}{Y(s)-N(s) Q(s)} e^{s L},
$$

where $N(s) \in R H_{\infty}$ and $D(s) \in R H_{\infty}$ are coprime factors of $G(s)$ on $R H_{\infty}$ satisfying

$$
G(s)=\frac{N(s)}{D(s)},
$$

$X(s) \in R H_{\infty}$ and $Y(s) \in R H_{\infty}$ are functions satisfying

$$
X(s) N(s)+Y(s) D(s)=1 .
$$

$Q(s) \in H_{\infty}$ is any function written by the form in

$$
\begin{aligned}
Q(s)= & \frac{-X(s) Q_{1}(s)+Y(s) Q_{2}(s) e^{-s L}}{D(s) Q_{1}(s)+N(s) Q_{2}(s) e^{-s L}} \\
& +\sum_{i=1}^{N} X(s) Q_{1}(s) q_{i}(s) e^{-s T_{i}} \\
& -\sum_{i=1}^{N} D(s) Q_{1}(s) q_{i}(s) e^{-s T_{i}} \\
& +\sum_{i=1}^{N} Y(s)\left(\bar{Q}_{i}(s)-Q_{2}(s)\right) q_{i}(s) e^{-s\left(T_{i}+L\right)} \\
& +\sum_{i=1}^{N} N(s)\left(\bar{Q}_{i}(s)-Q_{2}(s)\right) q_{i}(s) e^{-s\left(T_{i}+L\right)}
\end{aligned}
$$

where $Q_{1}(s) \neq 0, Q_{2}(s)$ and $\bar{Q}_{i}(s) \neq 0(i=1, \ldots, N)$ are any $R H_{\infty}$ functions.

Proof of Theorem 1 requires following lemma and theorem.

Lemma 1 Unity feedback control system in

$$
\left\{\begin{array}{l}
y(s)=G(s) u(s) \\
u(s)=-C(s) y(s)
\end{array}\right.
$$

is internally stable if and only if the controller $C(s)$ is written by

$$
C(s)=\frac{X(s)+D(s) Q(s)}{Y(s)-N(s) Q(s)}
$$


where $N(s) \in R H_{\infty}$ and $D(s) \in R H_{\infty}$ are coprime factors of $G(s)$ on $R H_{\infty}$ satisfying Eq. (8), $X(s) \in R H_{\infty}$ and $Y(s) \in R H_{\infty}$ are functions satisfying Eq. (9) and $Q(s) \in H_{\infty}$ is any function ${ }^{(2)}$.

Theorem 2 Assume that $C_{0}(s), C_{i}(s)(i=1, \ldots, N)$ and $q_{i}(s)(i=1, \ldots, N)$ in Eq. (3) are rational functions. The controller $C(s)$ in Eq. (3) stabilizes the plant $G(s) e^{-s L}$ if and only if the controller $\hat{C}(s)=C(s) e^{-s L}$ stabilizes $G(s)$.

(proof) Proof is obvious, because $C_{0}(s), C_{i}(s)(i=1, \ldots, N)$ and $q_{i}(s)(i=1, \ldots, N)$ in Eq. (3) are real rational functions.

Using Lemma 1 and Theorem 2, we show the proof of Theorem 1.

(proof) From Theorem 2, the parameterization of all stabilizing controllers $C(s)$ for the plant $G(s) e^{-s L}$ is equivalent to that of $\hat{C}(s)=C(s) e^{-s L}$ for $G(s)$.

First, necessity is shown. That is, we show that if $C(s)$ written as Eq. (3) stabilizes $G(s) e^{-s L}$, then the controller $C(s)=\hat{C}(s) e^{s L}$ and the free parameter $Q(s)$ are given by Eq. (7) and Eq. (10), respectively. From Lemma 1, the parameterization of all stabilizing controllers $\hat{C}(s)$ for the plant $G(s)$ is written by

$$
\hat{C}(s)=C(s) e^{-s L}=\frac{X(s)+D(s) Q(s)}{Y(s)-N(s) Q(s)} .
$$

This equation is equivalent to Eq. (7). Next, we show that if the controller $\hat{C}(s)=C(s) e^{-s L}$ stabilizes $G(s)$, then the free parameter $Q(s)$ in Eq. (7) is written by Eq. (10). Substituting $C(s)$ in Eq. (3) into Eq. (7), we have Eq. (10), where

$$
\begin{aligned}
& Q_{1}(s)=C_{0 d}(s) \bar{C}_{d}(s) \in R H_{\infty}, \\
& Q_{2}(s)=C_{0 n}(s) \bar{C}_{d}(s) \in R H_{\infty}
\end{aligned}
$$

and

$$
\bar{Q}_{i}(s)=C_{0 d}(s) \bar{C}_{i n}(s) \in R H_{\infty}(i=1, \ldots, N) .
$$

Here, $C_{0 n}(s) \in R H_{\infty}$ and $C_{0 d}(s) \in R H_{\infty}$ are coprime factors of $C_{0}(s)$ on $R H_{\infty}$ satisfying

$$
C_{0}(s)=\frac{C_{0 n}(s)}{C_{0 d}(s)}
$$

$\bar{C}_{i n}(s) \in R H_{\infty}(i=1, \ldots, N)$ and $\bar{C}_{d}(s) \in R H_{\infty}$ are defined by

$$
\bar{C}_{i n}(s)=C_{i n}(s) \prod_{i=1}^{j-1} C_{i d}(s) \prod_{i=j+1}^{N} C_{i d}(s) \quad(i, j=1, \ldots, N)
$$

and

$$
\bar{C}_{d}(s)=\prod_{i=1}^{N} C_{i d}(s)
$$

respectively. Here, $C_{i n}(s)(i=1, \ldots, N)$ and $C_{i d}(s)(i=1, \ldots, N)$ are coprime factors of $C_{i}(s)(i=1, \ldots, N)$ on $R H_{\infty}$ satisfying

$$
C_{i}(s)=\frac{C_{i n}(s)}{C_{i d}(s)}(i=1, \ldots, N) .
$$

From these equations, we have proved that if the controller $C(s)$ written as Eq. (3) stabilizes the control system in Eq. (1) then the free parameter $Q(s)$ in Eq. (7) is written by Eq. (10). From Eq. (14), Eq. (16) and $C_{i}(s)(i=1, \ldots, N)$ in Eq. (3) satisfies $C_{i}(s) \neq 0(i=1, \ldots, N)$, we can easily confirm that $Q_{1}(s) \neq 0$ and $\bar{Q}_{i} \neq 0(i=1, \ldots, N)$ are satisfied. Thus, necessity has been shown. 
Next sufficiency is shown. That is, we show that under assumptions of $Q_{1}(s) \neq 0$ and $\bar{Q}_{i}(s) \neq 0(i=1, \ldots, N)$, if the free parameter $Q(s)$ is given by Eq. (10), the controller $C(s)$ is written by Eq. (3). Substituting Eq. (10) into Eq. (7), we have

$$
C(s)=C_{0}(s)+\frac{\sum_{i=1}^{N} C_{i}(s) q_{i}(s) e^{-s T_{i}}}{1-\sum_{i=1}^{N} q_{i}(s) e^{-s T_{i}}},
$$

where $C_{0}(s)$ and $C_{i}(s)(i=1, \ldots, N)$ are denoted by

$$
C_{0}(s)=\frac{Q_{2}(s)}{Q_{1}(s)}
$$

and

$$
C_{i}(s)=\frac{\bar{Q}_{i}(s)}{Q_{1}(s)}(i=1, \ldots, N)
$$

respectively. From Eq. (23) and the assumption of $\bar{Q}_{i}(s) \neq 0(i=1, \ldots, N), C_{i}(s)(i=$ $1, \ldots, N)$ in Eq. (3) satisfies $C_{i}(s) \neq 0(i=1, \ldots, N)$. This implies that $C(s)$ in Eq. (7) works as a multi-period repetitive controller.

We have thus proved Theorem 1.

Remark 1 Note that even if the time-delay in the plant has uncertainty, the control system in Eq. (1) using the proposed parameterization in Eq. (7) is not necessarily unstable. The fact is confirmed as follows: The plant is assumed to be written by

$$
G(s) e^{-s(L+\Delta L)},
$$

where $\Delta L \neq 0$ is an uncertainty. Using the proposed parameterization in Eq. (7), the transfer function from the reference input $r(s)$ to the output $y(s)$ is written by

$$
\begin{aligned}
\frac{y(s)}{r(s)}= & \frac{N(s)\left\{Q_{2}(s)\left(1-\sum_{i=1}^{N} q_{i}(s) e^{-s T_{i}}\right)+\sum_{i=1}^{N} \bar{Q}_{i}(s) q_{i}(s) e^{-s T_{i}}\right\} e^{-s(L+\Delta L)}}{D(s) Q_{1}(s)\left(1-\sum_{i=1}^{N} q_{i}(s) e^{-s T_{i}}\right)} \\
& +N(s)\left\{Q_{2}(s)\left(1-\sum_{i=1}^{N} q_{i}(s) e^{-s T_{i}}\right)+\sum_{i=1}^{N} \bar{Q}_{i}(s) q_{i}(s) e^{-s T_{i}}\right\} e^{-s(L+\Delta L)}
\end{aligned}
$$

From Eq. (25), even if the time-delay in the plant includes uncertainty $\Delta L$, if the transfer function in Eq. (25) have no pole in the closed right half plain, the stability of the control system is guaranteed.

\section{Control characteristics}

In this section, we explain control characteristics of the control system in Eq. (1) using the proposed parameterization. In addition, roles of $Q_{1}(s), Q_{2}(s)$ and $\bar{Q}_{i}(s)(i=1, \ldots, N)$ in Eq. (10) are clarified.

From Theorem 1, $Q(s)$ in Eq. (10) must be included in $H_{\infty}$. Since $Q_{1}(s) \in R H_{\infty}$, $Q_{2}(s) \in R H_{\infty}$ and $\bar{Q}_{i}(s) \in R H_{\infty}(i=1, \ldots, N)$ in Eq. (10), if $1 /\left\{D(s) Q_{1}(s)+N(s) Q_{2}(s) e^{-s L}-\right.$ $\left.\sum_{i=1}^{N} D(s) Q_{1}(s) q_{i}(s) e^{-s T_{i}}+\sum_{i=1}^{N} N(s)\left(\bar{Q}_{i}(s)-Q_{2}(s)\right) q_{i}(s) e^{-s\left(T_{i}+L\right)}\right\} \in H_{\infty}$, then $Q(s)$ satisfies $Q(s) \in H_{\infty}$. That is, the role of $Q_{1}(s) \in R H_{\infty}, Q_{2}(s) \in R H_{\infty}$ and $\bar{Q}_{i}(s) \in R H_{\infty}(i=1, \ldots, N)$ is to assure the stability of the control system in Eq. (1).

Next, the input-output characteristic of the control system in Eq. (1) is shown. The transfer function $S(s)$ from the periodic reference input $r(s)$ to the error $e(s)=r(s)-y(s)$ of 
the control system in Eq. (1) is written by

$$
\begin{aligned}
S(s)= & \frac{D(s) Q_{1}(s)\left(1-\sum_{i=1}^{N} q_{i}(s) e^{-s T_{i}}\right)}{D(s) Q_{1}(s)\left(1-\sum_{i=1}^{N} q_{i}(s) e^{-s T_{i}}\right)} \\
& \frac{+N(s)\left\{Q_{2}(s)\left(1-\sum_{i=1}^{N} q_{i}(s) e^{-s T_{i}}\right)+\sum_{i=1}^{N} \bar{Q}_{i}(s) q_{i}(s) e^{-s T_{i}}\right\} e^{-s L}}{} .
\end{aligned}
$$

Since $q_{i}(s)(i=1, \ldots, N)$ are settled beforehand to satisfy Eq. (5), the output $y(s)$ follows the periodic reference input $r(s)$ with small steady state error. If the gain of $\bar{Q}_{i}(s)(i=1, \ldots, N)$ increase, then steady state error decrease. That is, the role of $q_{i}(s)(i=1, \ldots, N)$ is to specify the input-output characteristic of the control system in Eq. (1). In addition, $\bar{Q}_{i}(s)(i=1, \ldots, N)$ play an auxiliary role to specify the input-output characteristic of the control system in Eq. (1).

Next, the disturbance attenuation characteristic of the control system in Eq. (1) is shown. The transfer function from the disturbance $d(s)$ to the output $y(s)$ of the control system in Eq. (1) is written by Eq. (26). From Eq. (5) and Eq. (26), for the disturbance $d(s)$ with same frequency components $\omega_{k}(k=0,1, \ldots, n)$ of the periodic reference input $r(s), S\left(j \omega_{k}\right)$ satisfy $S\left(j \omega_{k}\right) \simeq 0(\forall k=0,1, \ldots, n)$ independent from $Q_{1}(s)$ and $Q_{2}(s)$. This implies that the disturbance with same frequency components $\omega_{k}(k=0,1, \ldots, n)$ of the periodic reference input $r(s)$ is attenuated effectively. That is, the role of $q_{i}(s)(i=1, \ldots, N)$ is to specify the disturbance attenuation characteristic for the disturbance with same frequency components $\omega_{k}(k=0,1, \ldots, n)$ of the periodic reference input $r(s)$. For the frequency component $\omega_{d}$ of the disturbance $d(s)$ that is different from that of the periodic reference input $r(s)$, that is $\omega_{d} \neq \omega_{k}(\forall k=0,1, \ldots, n)$, even if

$$
1-\sum_{i=1}^{N} q_{i}\left(j \omega_{d}\right) \simeq 0,
$$

the disturbance $d(s)$ cannot be attenuated, because

$$
e^{-j \omega_{d} T_{i}} \neq 1 \quad(i=1, \ldots, N)
$$

and

$$
1-\sum_{i=1}^{N} q_{i}\left(j \omega_{d}\right) e^{-j \omega_{d} T_{i}} \neq 0 .
$$

In order to attenuate this frequency component, if $Q_{1}(s)$ is settled satisfying

$$
Q_{1}\left(j \omega_{d}\right) \simeq 0
$$

then the disturbance $d(s)$ is attenuated effectively. That is, the role of $Q_{1}(s)$ is to specify the disturbance attenuation characteristic for the disturbance with frequency component $\omega_{d} \neq$ $\omega_{k}(\forall k=0,1, \ldots, n)$.

In summary, the role of $Q_{1}(s), Q_{2}(s)$ and $\bar{Q}_{i}(s)(i=1, \ldots, N)$ is to assure the stability of the control system in Eq. (1) by satisfying $Q(s) \in H_{\infty}$. The role of $q_{i}(s)(i=1, \ldots, N)$ is to specify the input-output characteristic of the control system in Eq. (1) and to specify the disturbance attenuation characteristic for disturbance $d(s)$ with same frequency components $\omega_{k}(k=0,1, \ldots, n)$ of the periodic reference input $r(s)$. The role of $\bar{Q}_{i}(s)(i=1, \ldots, N)$ play an auxiliary role to specify the input-output characteristic of the control system in Eq. (1). The role of $Q_{1}(s)$ is to specify the disturbance attenuation characteristic for disturbance $d(s)$ with frequency components $\omega_{d} \neq \omega_{k}(\forall k=0,1, \ldots, n)$.

\section{Design procedure}

In this section, a design procedure of stabilizing multi-period repetitive controller for time-delay plants with the specified input-output characteristic is presented. 
A design procedure of stabilizing multi-period repetitive controllers satisfying Theorem 1 is summarized as follows:

\section{$\underline{\text { Procedure }}$}

(1) Low-pass filters $q_{i}(s) \in R H_{\infty}(i=1, \ldots, N)$ in Eq. (10) are settled so that for the frequency component $\omega_{k}(k=0,1, \ldots, n)$ of the periodic reference input $r(s)$,

$$
1-q\left(j \omega_{k}\right) \simeq 0
$$

is satisfied.

( 2 ) Obtain coprime factors $N(s) \in R H_{\infty}$ and $D(s) \in R H_{\infty}$ of $G(s)$ satisfying Eq. (8).

( 3 ) $X(s) \in R H_{\infty}$ and $Y(s) \in R H_{\infty}$ are settled satisfying Eq. (9).

(4) $Q_{1}(s) \in R H_{\infty}$ is settled so that for the frequency component $\omega_{d}$ of the disturbance $d(s), Q_{1}\left(j \omega_{d}\right) \simeq 0$ is satisfied. In order to design $Q_{1}(s)$ to hold $Q_{1}\left(j \omega_{d}\right) \simeq 0, Q_{1}(s)$ is settled by

$$
Q_{1}(s)=1-\frac{1}{(1+s \tau)^{\alpha}} \in R H_{\infty},
$$

where $\alpha$ is an arbitrary positive integer to make $Q_{1}(s)$ proper and $\tau \in R$ is a sufficiently small positive real number satisfying

$$
1-\frac{1}{\left(1+j \omega_{d} \tau\right)^{\alpha}} \simeq 0 \text {. }
$$

( 5$) \quad Q_{2}(s) \in R H_{\infty}$ and $\bar{Q}_{i}(s) \in R H_{\infty}(i=1, \ldots, N)$ are settled so that $Q(s)$ in Eq. (10) is included in $H_{\infty}$.

\section{Numerical example}

In this section, a numerical example is shown to illustrate the effectiveness of the proposed parameterization. Consider the problem to obtain the parameterization of all stabilizing multi-period repetitive controllers with the specified input-output characteristic for the timedelay plant $G(s) e^{-s L}$ written by

$$
G(s) e^{-s L}=\frac{s+130}{s^{2}+18 s+80} e^{-2 s},
$$

where

$$
G(s)=\frac{s+130}{s^{2}+18 s+80}
$$

and

$$
L=2 \text {, }
$$

such that the output $y(t)$ follows the periodic reference input $r(t)$ with period $T=2$ [sec]. $N$ in Eq. (3) is set as $N=3$. Using the method of Ref. (12), $T_{i}(i=1,2,3)$ and $q_{i}(s)(i=1,2,3)$ in Eq. (3) are settled by

$$
\begin{aligned}
& T_{1}=1.9900, \\
& T_{2}=4.0704, \\
& T_{3}=5.6447, \\
& q_{1}(s)=\frac{1}{0.01 s+1}, \\
& q_{2}(s)=\frac{1}{0.01 s+1} \cdot \frac{0.0027 s\left(s^{2}+\pi^{2}\right)}{(s+1)^{3}}
\end{aligned}
$$

and

$$
q_{3}(s)=\frac{1}{0.01 s+1} \cdot \frac{0.0027 s\left(s^{2}+\pi^{2}\right)}{(s+1)^{3}} \cdot \frac{1.8662\left(s^{2}+(2 \pi)^{2}\right)}{s^{2}+s+180},
$$


respectively.

A pair of coprime factors $N(s) \in R H_{\infty}$ and $D(s) \in R H_{\infty}$ of $G(s)$ in Eq. (35) satisfying Eq. (8) are given by

$$
N(s)=G(s)=\frac{s+130}{s^{2}+18 s+80} \in R H_{\infty}
$$

and

$$
D(s)=1 \in R H_{\infty} .
$$

$X(s) \in R H_{\infty}$ and $Y(s) \in R H_{\infty}$ satisfying Eq. (9) are settled by

$$
X(s)=0 \in R H_{\infty}
$$

and

$$
Y(s)=1 \in R H_{\infty} .
$$

According to Theorem 1, the parameterization of all stabilizing multi-period repetitive controllers for the time-delay plant with the specified input-output characteristic is written by Eq. (7) and Eq. (10).

In order to satisfy $Q(s) \in H_{\infty}, Q_{1}(s), Q_{2}(s)$ and $\bar{Q}_{i}(s)(i=1,2,3)$ in Eq. (10) are settled by

$$
\begin{aligned}
& Q_{1}(s)=1-\frac{1}{0.05 s+1} \in R H_{\infty}, \\
& Q_{2}(s)=\frac{5(s+0.3)(s+25)(s+300)}{(s+100)^{3}} \in R H_{\infty}
\end{aligned}
$$

and

$$
\bar{Q}_{i}(s)=\frac{5(s+0.3)(s+25)(s+300)}{(s+100)^{3}}(i=1,2,3) \in R H_{\infty},
$$

respectively.

From the Nyquist theorem, $Q_{1}(s) \in R H_{\infty}, Q_{2}(s) \in R H_{\infty}$ and $\bar{Q}_{i}(s) \in R H_{\infty}(i=1,2,3)$, if the Nyquist plot of $D(s) Q_{1}(s)+N(s) Q_{2}(s) e^{-s L}-\Sigma_{i=1}^{N} D(s) Q_{1}(s) q_{i}(s) e^{-s T_{i}}+\Sigma_{i=1}^{N} N(s)$ $\left(\bar{Q}_{i}(s)-Q_{2}(s)\right) q_{i}(s) e^{-s\left(T_{i}+L\right)}$ does not encircle the origin, then $Q(s) \in H_{\infty}$ holds true. Using $Q_{1}(s), Q_{2}(s)$ and $\bar{Q}_{i}(s)(i=1,2,3)$ in Eqs. (47) (49), the Nyquist plot of $D(s) Q_{1}(s)+$ $N(s) Q_{2}(s) e^{-s L}-\Sigma_{i=1}^{N} D(s) Q_{1}(s) q_{i}(s) e^{-s T_{i}}+\Sigma_{i=1}^{N} N(s)\left(\bar{Q}_{i}(s)-Q_{2}(s)\right) q_{i}(s) e^{-s\left(T_{i}+L\right)}$ is shown in Fig. 1. From Fig. 1, since the Nyquist plot of $D(s) Q_{1}(s)+N(s) Q_{2}(s) e^{-s L}$

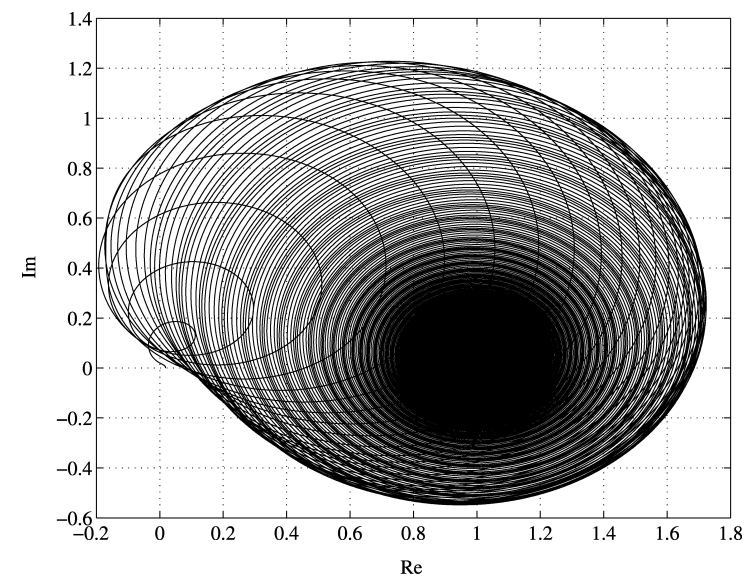

Fig. $1 \quad$ Nyquist plot of $D(s) Q_{1}(s)+N(s) Q_{2}(s) e^{-s L}-\Sigma_{i=1}^{N} D(s) Q_{1}(s) q_{i}(s) e^{-s T_{i}}+$ $\sum_{i=1}^{N} N(s)\left(\bar{Q}_{i}(s)-Q_{2}(s)\right) q_{i}(s) e^{-s\left(T_{i}+L\right)}$ 
$-\sum_{i=1}^{N} D(s) Q_{1}(s) q_{i}(s) e^{-s T_{i}}+\sum_{i=1}^{N} N(s)\left(\bar{Q}_{i}(s)-Q_{2}(s)\right) q_{i}(s) e^{-s\left(T_{i}+L\right)}$ does not encircle the origin, we find that $Q(s)$ in Eq. (10) is included in $H_{\infty}$.

Using above-mentioned parameters, we have a stabilizing multi-period repetitive controller for the time-delay plant with the specified input-output characteristic. When the designed multi-period repetitive controller $C(s)$ is used, the response of the output $y(t)$ of control system in Eq. (1) with the plant in Eq. (34) for the periodic reference input $r(t)=\sin (\pi t)$ is shown in Fig. 2. Here, the broken line shows the response of the periodic reference input $r(t)$

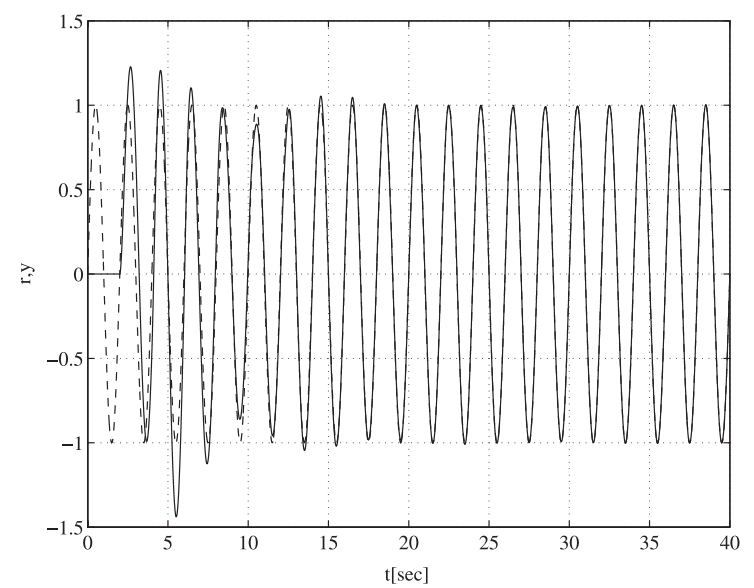

Fig. 2 Response of the output $y(t)$ for the periodic reference input $r(t)=\sin (\pi t)$

and the solid line shows that of the output $y(t)$. Figure 2 shows that the output $y(t)$ follows the periodic reference input $r(t)$ with small steady state error.

Next, using the designed stabilizing multi-period repetitive controller $C(s)$, the disturbance attenuation characteristic is shown. The response of the output $y(t)$ for the disturbance $d(t)=\sin (\pi t)$ of which the frequency component is equivalent to that of the periodic reference input $r(t)$ is shown in Fig. 3. Here, the broken line shows the response of the disturbance $d(t)$

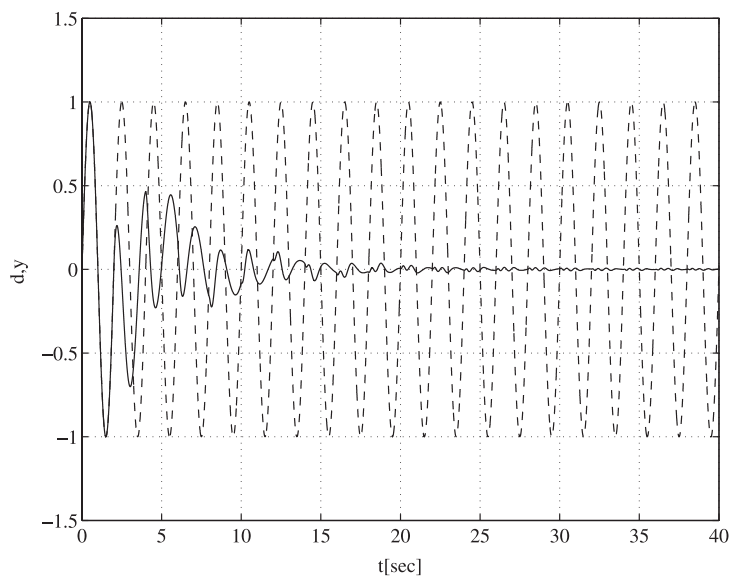

Fig. 3 Response of the output $y(t)$ for the disturbance $d(t)=\sin (\pi t)$

and the solid line shows that of the output $y(t)$. Figure 3 shows that the disturbance $d(t)$ is attenuated effectively. Finally, the response of the output $y(t)$ for the disturbance $d(t)=\sin (2 \pi t / 43)$ of which the frequency component is different from that of the periodic reference input $r(t)$ is shown in Fig. 4. Here, the broken line shows the response of the disturbance $d(t)$ and the solid line shows that of the output $y(t)$. Figure 4 shows that the disturbance $d(t)$ is attenuated.

When the time-delay includes uncertainty $\Delta L=-0.1$, that is, the plant is given by

$$
\begin{aligned}
G(s) e^{-s(L+\Delta L)} & =\frac{s+130}{s^{2}+18 s+80} e^{-(2-0.1) s} \\
& =\frac{s+130}{s^{2}+18 s+80} e^{-1.9 s},
\end{aligned}
$$




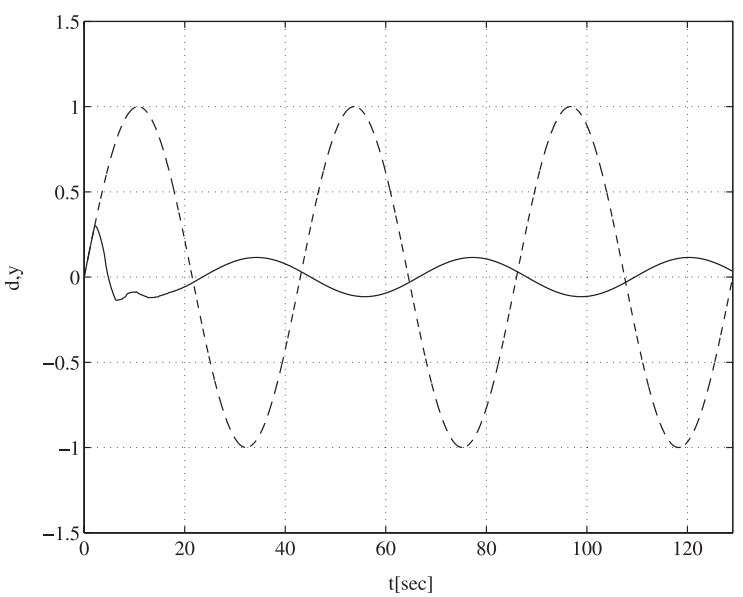

Fig. 4 Response of the output $y(t)$ for the disturbance $d(t)=\sin \left(\frac{2}{43} \pi t\right)$

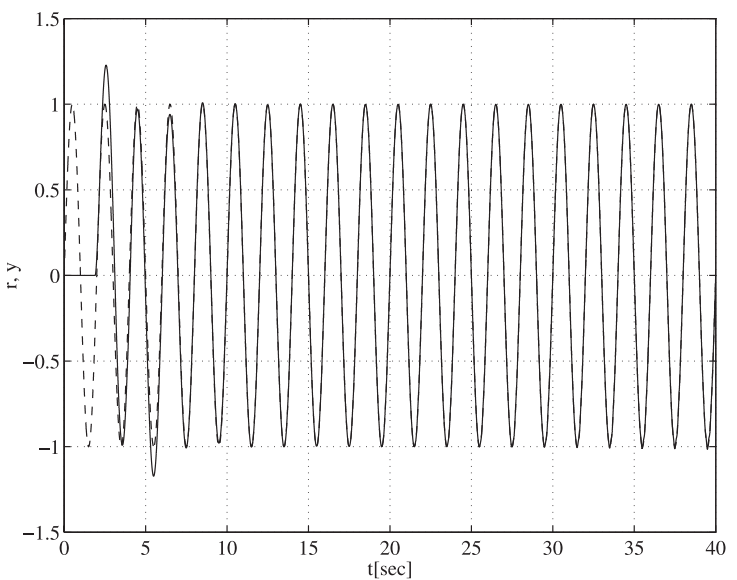

Fig. 5 Response of the output $y(t)$ for the periodic reference input $r(t)=\sin (\pi t)$ when the uncertainty $\Delta L=-0.1$ exists

the response of the output $y(t)$ for the periodic reference input $r(t)=\sin (\pi t)$ is shown in Fig. 5 . Here, the broken line shows the response of the periodic reference input $r(t)$ and the solid line shows that of the output $y(t)$. Figure 5 shows that the control system in Eq. (1) is stable even if the uncertainty $\Delta L$ exists.

In order to show that the effectiveness of the proposed method, the difference was clarified by comparison with the response using the parameterization of all stabilizing multi-period repetitive controllers for time-delay systems in Ref. (9). According to Ref. (9), the parameterization of all stabilizing multi-period repetitive controllers for time-delay systems is given by

$$
C(s)=\frac{X(s)+D(s) \hat{Q}(s)}{Y(s)-N(s) \hat{Q}(s)} e^{s L},
$$

where $Q(s) \in H_{\infty}$ is any function written by the form in

$$
Q(s)=\frac{Q_{n 1}(s)+Q_{n 2}(s) e^{-s L}+\sum_{i=1}^{N} Q_{n 3 i}(s) e^{-s T_{i}}+\sum_{i=1}^{N} Q_{n 4 i}(s) e^{-s\left(T_{i}+L\right)}}{Q_{d 1}(s)+Q_{d 2}(s) e^{-s L}+\sum_{i=1}^{N} Q_{d 3 i}(s) e^{-s T_{i}}+\sum_{i=1}^{N} Q_{d 4 i}(s) e^{-s\left(T_{i}+L\right)}},
$$

where $Q_{n 1}(s), Q_{n 2}(s), Q_{n 3 i}(s)(i=1, \ldots, N), Q_{n 4 i}(s)(i=1, \ldots, N), Q_{d 1}(s) \neq 0, Q_{d 2}(s)$, $Q_{d 3 i}(s)(i=1, \ldots, N)$ and $Q_{d 4 i}(s)(i=1, \ldots, N)$ are any $R H_{\infty}$ functions satisfying

$$
\sum_{i=1}^{N}\left(-\frac{Y(0) Q_{d 3 i}(0)-N(0) Q_{n 3 i}(0)}{Y(0) Q_{d 1}(0)-N(0) Q_{n 1}(0)}\right)=1
$$




$$
\begin{aligned}
& X(s) Q_{d 1}(s)+D(s) Q_{n 1}(s)=0, \\
& Y(s) Q_{d 2}(s)-N(s) Q_{n 2}(s)=0, \\
& X(s) Q_{d 3 i}(s)+D(s) Q_{n 3 i}(s)=0 \quad(i=1, \ldots, N), \\
& Y(s) Q_{d 4 i}(s)-N(s) Q_{n 4 i}(s)=0 \quad(i=1, \ldots, N)
\end{aligned}
$$

and

$$
\begin{aligned}
& \frac{\left(X(s) Q_{d 4 i}(s)+D(s) Q_{n 4 i}(s)\right)\left(Y(s) Q_{d 1}(s)-N(s) Q_{n 1}(s)\right)}{\left(Y(s) Q_{d 1}(s)-N(s) Q_{n 1}(s)\right)^{2}} \\
& \frac{-\left(X(s) Q_{d 2}(s)+D(s) Q_{n 2}(s)\right)\left(Y(s) Q_{d 3 i}(s)-N(s) Q_{n 3 i}(s)\right)}{} \neq 0 \quad(i=1, \ldots, N) .
\end{aligned}
$$

Substituting Eq. (52) into Eq. (51), we have Eq. (3), where $q_{i}(s)(i=1, \ldots, N)$ is denoted by

$$
q_{i}(s)=-\frac{Y(s) Q_{d 3 i}(s)-N(s) Q_{n 3 i}(s)}{Y(s) Q_{d 1}(s)-N(s) Q_{n 1}(s)}(i=1, \ldots, N) .
$$

Using the parameterization in Eq. (51), we design a stabilizing multi-period repetitive controller for the plant in Eq. (34). $N$ in Eq. (3) is $N=3$. The period $T$ of the reference input is $T=2$ [sec] and $T_{i}=T \cdot i(i=1,2,3) . N(s) \in R H_{\infty}, D(s) \in R H_{\infty}, X(s) \in R H_{\infty}$ and $Y(s) \in R H_{\infty}$ are settled by Eq. (43), Eq. (44), Eq. (45) and Eq. (46), respectively.

In order for the free parameter $Q(s)$ to satisfy $Q(s) \in H_{\infty}$ and Eq. (53) Eq. (58), $Q_{n 1}(s)$, $Q_{n 2}(s), Q_{n 3 i}(s)(i=1, \ldots, N), Q_{n 4 i}(s)(i=1, \ldots, N), Q_{d 1}(s) \neq 0, Q_{d 2}(s), Q_{d 3 i}(s)(i=1, \ldots, N)$ and $Q_{d 4 i}(s)(i=1, \ldots, N)$ are settled as follows : From Eq. (43) Eq. (46), Eq. (54) Eq. (58) and Eq. (59), we have

$$
\begin{aligned}
& Q_{n 1}(s)=0 \in R H_{\infty}, \\
& Q_{d 2}(s)=G(s) Q_{n 2} \in R H_{\infty}, \\
& Q_{n 3 i}(s)=0 \in R H_{\infty}, \\
& q_{i}(s)=-\frac{Q_{d 3 i}(s)}{Q_{d 1}(s)} \in R H_{\infty}(i=1,2,3)
\end{aligned}
$$

and

$$
Q_{d 4 i}=G(s) Q_{n 4 i}(s) \in R H_{\infty}(i=1,2,3) .
$$

$Q_{d 1}(s), Q_{n 2}(s), Q_{d 3 i}(s)$ and $Q_{n 4 i}(s)$ are used to make $Q(s) \in H_{\infty}$. Since $Q_{n 2}(s)$ and $Q_{n 4 i}(s)$ $(i=1, \ldots, N)$ are included in $R H_{\infty}$, if $1 /\left\{Q_{d 1}(s)+Q_{d 2}(s) e^{-s L}+\sum_{i=1}^{N} Q_{d 3 i}(s) e^{-s T_{i}}+\right.$ $\left.\sum_{i=1}^{N} Q_{d 4 i}(s) e^{-s\left(T_{i}+L\right)}\right\} \in H_{\infty}$ then $Q(s) \in H_{\infty}$ holds true. From nyquist theorem, if the nyquist plot of $Q_{d 1}(s)+Q_{d 2}(s) e^{-s L}+\sum_{i=1}^{N} Q_{d 3 i}(s) e^{-s T_{i}}+\sum_{i=1}^{N} Q_{d 4 i}(s) e^{-s\left(T_{i}+L\right)}$ dose not encircle the origin, then $1 /\left\{Q_{d 1}(s)+Q_{d 2}(s) e^{-s L}+\sum_{i=1}^{N} Q_{d 3 i}(s) e^{-s T_{i}}+\sum_{i=1}^{N} Q_{d 4 i}(s) e^{-s\left(T_{i}+L\right)}\right\} \in H_{\infty}$ holds true. When $Q_{d 1}(s), Q_{n 2}(s), Q_{d 3 i}(s)$ and $Q_{n 4 i}(s)$ are given by

$$
\begin{aligned}
& Q_{d 1}(s)=\frac{0.005 s+1}{4 s+1}, \\
& Q_{n 2}(s)=\frac{2 s^{2}+36 s+160}{14 s^{2}+1821 s+130}, \\
& Q_{d 31}(s)=-\frac{0.005 s+1}{2\left(0.02 s^{2}+4.005 s+1\right)}, \\
& Q_{d 32}(s)=-\frac{0.005 s+1}{3\left(0.02 s^{2}+4.005 s+1\right)}
\end{aligned}
$$




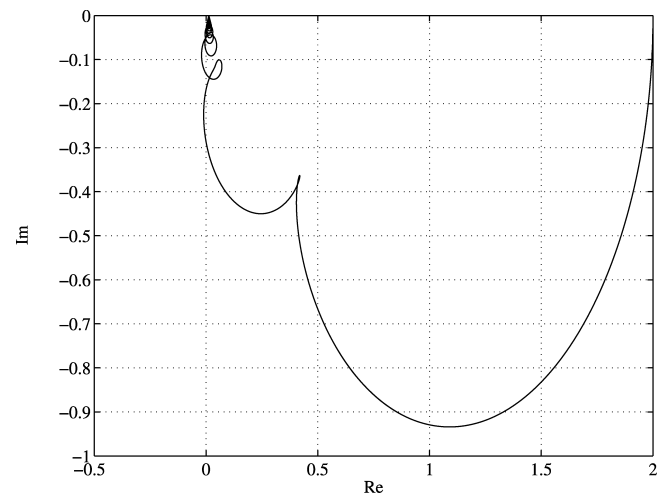

Fig. 6 The nyquist plot of $Q_{d 1}(s)+Q_{d 2}(s) e^{-s L}+\Sigma_{i=1}^{N} Q_{d 3 i}(s) e^{-s T_{i}}+\Sigma_{i=1}^{N} Q_{d 4 i}(s) e^{-s\left(T_{i}+L\right)}$

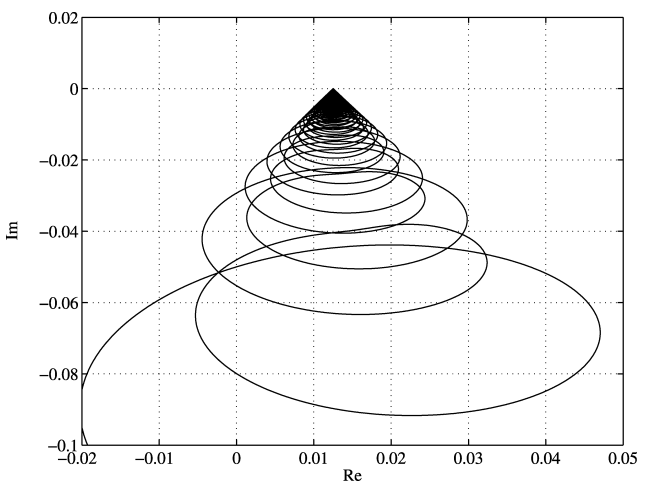

Fig. 7 A specially magnified detail drawing showing the origin of the nyquist plot of $Q_{d 1}(s)+Q_{d 2}(s) e^{-s L}+\Sigma_{i=1}^{N} Q_{d 3 i}(s) e^{-s T_{i}}+\Sigma_{i=1}^{N} Q_{d 4 i}(s) e^{-s\left(T_{i}+L\right)}$

and

$$
Q_{d 33}(s)=-\frac{0.005 s+1}{6\left(0.02 s^{2}+4.005 s+1\right)},
$$

$$
Q_{n 4 i}(s)=0,
$$

the nyquist plot of the denominator of $Q(s)$ is shown in Fig. 6. A specially magnified detail drawing showing the origin of Fig. 6 is shown in Fig. 7. Fig. 6 and Fig. 7 show that the nyquist plot of $1 /\left\{Q_{d 1}(s)+Q_{d 2}(s) e^{-s L}+\sum_{i=1}^{N} Q_{d 3 i}(s) e^{-s T_{i}}+\sum_{i=1}^{N} Q_{d 4 i}(s) e^{-s\left(T_{i}+L\right)}\right\}$ dose not encircle the origin. Since the nyquist plot of the denominator of $Q(s)$ dose not encircle the origin, $Q(s) \in H_{\infty}$ holds true.

Using the obtained multi-period repetitive controller, the response of the output $y(t)$ of control system in Eq. (1) with the plant in Eq. (34) for the periodic reference input $r(t)=$ $\sin (\pi t)$ is shown in Fig. 8. Here, the broken line shows the response of the periodic reference input $r(t)$ and the solid line shows that of the output $y(t)$. Figure 8 shows that the output $y(t)$ follows the periodic reference input $r(t)$ with small steady state error.

Next, the response of the output $y(t)$ for the disturbance $d(t)=\sin (\pi t)$ of which the frequency component is equivalent to that of the periodic reference input $r(t)$ is shown in Fig. 9. Here, the broken line shows the response of the disturbance $d(t)$ and the solid line shows that of the output $y(t)$. Figure 9 shows that the disturbance $d(t)$ is attenuated effectively. Finally, the response of the output $y(t)$ for the disturbance $d(t)=\sin (2 \pi t / 43)$ of which the frequency component is different from that of the periodic reference input $r(t)$ is shown in Fig. 10. Here, the broken line shows the response of the disturbance $d(t)$ and the solid line shows that of the output $y(t)$. Figure 10 shows that using the method in Ref. (9), the disturbance attenuation characteristic for disturbance with frequency components $\omega_{d} \neq \omega_{k}(\forall k=0,1, \ldots, n)$ is not so good. The comparison of Fig. 2, Fig. 3 and Fig. 4 with Fig. 8, Fig. 9 and Fig. 10 shows that the proposed multi-period repetitive controller $C(s)$ in Eq. (7) with $Q(s)$ in Eq. (10) is more 


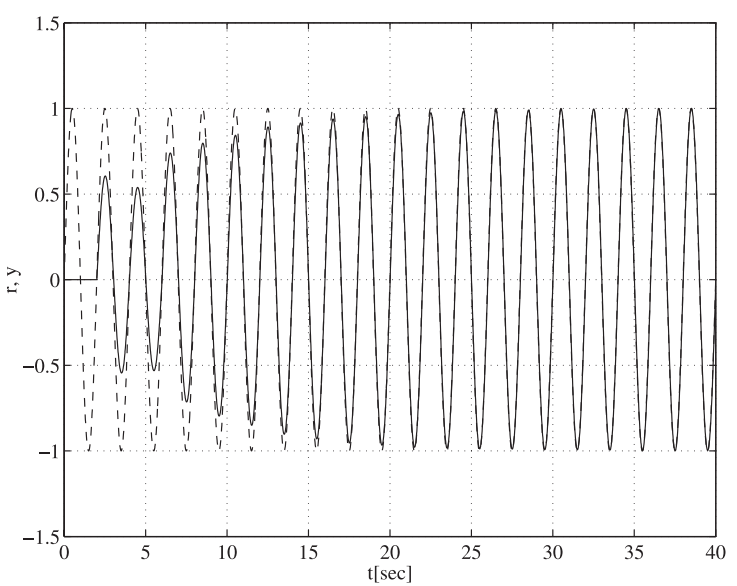

Fig. 8 Response of the output $y(t)$ for the periodic reference input $r(t)=\sin (\pi t)$ using the method in Ref. (9)

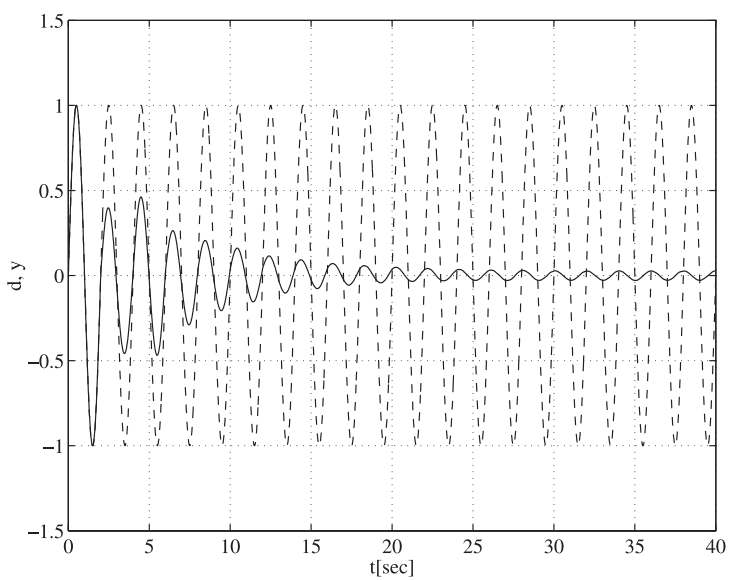

Fig. 9 Response of the output $y(t)$ for the disturbance $d(t)=\sin (\pi t)$ using the method in Ref. (9)

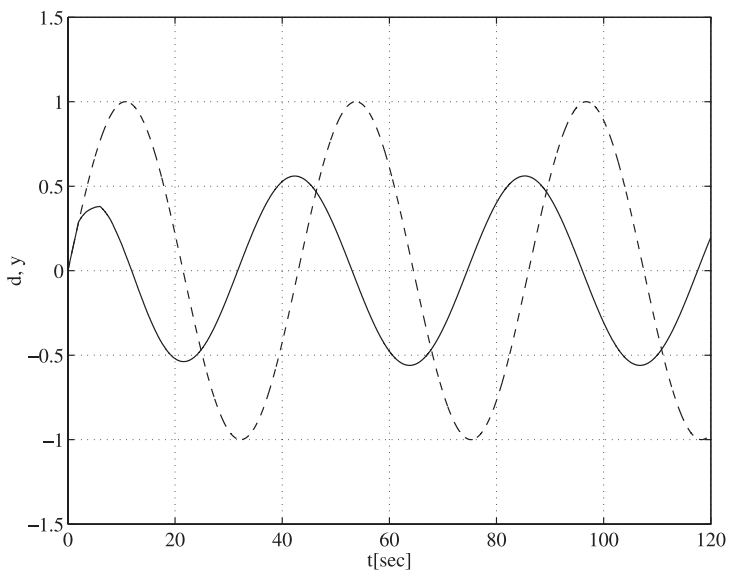

Fig. 10 Response of the output $y(t)$ for the disturbance $d(t)=\sin \left(\frac{2}{43} \pi t\right)$ using the method in Ref. (9)

effective controller than the multi-period repetitive controller $C(s)$ in Eq. (51) with $Q(s)$ in Eq. (52) proposed by Ref. (9). Especially, using the proposed method, the disturbance attenuation characteristic for disturbance with frequency components $\omega_{d} \neq \omega_{k}(\forall k=0,1, \ldots, n)$ is better than that using the method in Ref. (9).

In this way, it is shown that using the obtained parameterization, we can easily design a stabilizing multi-period repetitive controller. 


\section{Conclusions}

In this paper, we proposed the parameterization of all stabilizing multi-period repetitive controllers for time-delay plants with the specified input-output characteristic. Control characteristics of stabilizing multi-period repetitive control systems were also presented. Finally, a numerical example was shown to illustrate the effectiveness of the proposed parameterization. From Eq. (59), using the method in Ref. (9), we cannot settle the input-output characteristic easily, because we must settle four free parameters in order to specify the input-output characteristic. Proposed parameterization solved this problem by specifying low-pass filters beforehand. In addition, from Eq. (10) and Eq. (52), the number of free parameters in the proposed parameterization which must be settled is very small as compared with that in the parameterization of all stabilizing multi-period repetitive controllers for time-delay systems in Ref. (9). This fact means that using the proposed parameterization, we can design multiperiod repetitive control system easily than the method in Ref. (9). Because the multi-period repetitive control system using the proposed parameterization has merits, such as the stability of the control system with time-delay is guaranteed and the input-output characteristic is easily specified, practical applications for real time-delay system, for example, remote control and temperature control, are expected.

\section{References}

( 1 ) Youla, D.C., Jabr, H. and Bongiorno, J.J., Modern Wiener-Hopf design of optimal controllers. Part I, IEEE Trans. on Automatic Control, Vol.AC-21(1976), pp.3-13.

( 2 ) Vidyasagar, M., Control System Synthesis - A factorization approach -, MIT Press, (1985).

( 3 ) Hara, S. and Yamamoto, Y., Stability of Multivariable Repetitive Control Systems - Stability Condition and Class of Stabilizing Controllers, Trans. of the Society of Instrument and Control Engineers, Vol.22, No.12(1986), pp.1256-1261.

( 4 ) Katoh, H. and Funahashi, Y., A Design Method of Repetitive Controllers, Trans. of the Society of Instrument and Control Engineers, Vol.32, No.12(1996), pp.1601-1605.

( 5 ) Yamada, K. and Okuyama, T., A parameterization of all stabilizing repetitive controllers for linear minimum phase systems, Trans. of the Society of Instrument and Control Engineers, Vol.36, No.4(2000), pp.328-334.

( 6 ) Yamada, K., Satoh, K., Iida, N. and Okuyama, T., Control structure of all stabilizing repetitive controllers for the non-minimum phase systems, Proceeding of the 4th Asian Control Conference, (2002), pp.753-758.

( 7 ) Gotou, M., Matubayasi, S., Miyazaki, F., Kawamura, S. and Arimoto, S., A Robust Servo System with an Iterative Learning Compensator and Proposal of Multi-Period Learning Compensator, System and Control, Vol.31(1987), pp.376-374. (in Japanese)

( 8 ) Yamada, K., Satoh, K. and Arakawa, T., The parametrization of all stabilizing multiperiod repetitive controllers, The International Conference on Cybernetics and Information Technologies, Systems and Applications 2004 Proceedings Vol.II(2004), pp.358363, Orlando, U.S.A.

( 9 ) Satoh, K. and Yamada, K., The parametrization of all stabilizing multi-period repetitive controllers for time-delay systems, Proceedings of The 2005 Electrical Engineering/Electronics, Computer, Telecommunication, and Information Technology (ECTI) International Conference, Vol.I of II(2005), pp.243-346, Pattaya, Thailand.

(10) Sugimoto, H. and Washida, K., A Production of Modified Repetitive Control with Corrected Dead Time, Trans. Soc. Instrument and Control Engineers, Vol. 34(1998), pp. 645-647.

(11) Sugimoto, H. and Washida, K., A Design Method for Modified Repetitive Control with Corrected Dead Time, Trans. Soc. Instrument and Control Engineers, Vol. 34(1998), pp. 761-768.

(12) Okuyama, T., Yamada, K. and Satoh, K., A Design Method for Repetitive Control Sys- 
tems with a Multi-Period Repetitive Compensator, Theoretical and Applied Mechanics Japan, Vol. 51(2002), pp. 161-167.

(13) Yamada, K., Satoh, K., Arakawa, T. and Okuyama, T., A Design Method for Repetitive Control Systems with Multi-Period Repetitive Compensator, Trans. Japan Soc. Mechanical Engineers, Vol. 69, No. 686(2003), pp. 2691-2699.

(14) Yamada, K., Satoh, K. and Arakawa, T., A Design Method for Multiperiod Repetitive Controllers (Design Method Using the Parameterization of all Multiperiod Repetitive Controllers), Trans. Japan Soc. Mechanical Engineers, Vol. 71, No. 710C(2005), pp. 2945-2952.

(15) Broberg, H.L. and Molyet, R.G., A new approach to phase cancellation in repetitive control, Proc. of the 29th IEEE IAS, pp. 1766-1770, (1994).

(16) Steinbuch M., Repetitive Control for Systems with Uncertain Period-time, Automatica, Vol. 38(2002), pp. 2103-2109. 